\title{
On Roots of Polynomials and Algebraically Closed Fields
}

\author{
Christoph Schwarzweller \\ Institute of Informatics \\ University of Gdańsk \\ Poland
}

\begin{abstract}
Summary. In this article we further extend the algebraic theory of polynomial rings in Mizar 1, 2, 3. We deal with roots and multiple roots of polynomials and show that both the real numbers and finite domains are not algebraically closed [5, 7]. We also prove the identity theorem for polynomials and that the number of multiple roots is bounded by the polynomial's degree [4, 6].
\end{abstract}

MSC: 13A05 13B25 03B35

Keywords: commutative algebra; polynomials; algebraic closed fields

MML identifier: RING_5, version: 8.1.06 5.44.1305

\section{Preliminaries}

From now on $n$ denotes a natural number.

Note that there exists a natural number which is non trivial and non prime. Now we state the proposition:

(1) Let us consider an even natural number $n$, and an element $x$ of $\mathbb{R}_{\mathrm{F}}$. Then $x^{n} \geqslant 0 \mathbb{R}_{\mathrm{F}}$.

PROOF: Define $\mathcal{P}$ [natural number] $\equiv x^{2 \cdot \$_{1}} \geqslant 0_{\mathbb{R}_{\mathrm{F}}}$. For every element $x$ of $\mathbb{R}_{\mathrm{F}}, x^{2} \geqslant 0_{\mathbb{R}_{\mathrm{F}}}$. For every natural number $k, \mathcal{P}[k]$.

Let us consider a ring $R$ and an element $a$ of $R$. Now we state the propositions:

(2) $2 \star a=a+a$.

(3) $a^{2}=a \cdot a$. 
Let $F$ be a field and $a$ be an element of $F$. Note that $\frac{a}{1_{F}}$ reduces to $a$. One can check that $\mathbb{Z} / 2$ is non trivial and almost left invertible.

Let $n$ be a non trivial, non prime natural number. Note that $\mathbb{Z} / n$ is non integral domain-like and $\mathbb{Z} / 6$ is non degenerated.

\section{Some More Properties of Polynomials}

Let $R$ be a non degenerated ring. Observe that every non zero polynomial over $R$ is non-zero and every polynomial over $R$ which is monic is also non zero.

Let $p$ be a non zero polynomial over $R$. One can check that $\operatorname{deg} p$ is natural.

Let $R$ be a ring, $p$ be a zero polynomial over $R$, and $q$ be a polynomial over $R$. Let us observe that $p * q$ is zero and $q * p$ is zero.

Let us observe that $p+q$ reduces to $q$ and $q+p$ reduces to $q$.

Let $p$ be a polynomial over $R$. One can check that $p * \mathbf{0} . R$ reduces to $\mathbf{0} . R$ and $p * 1 . R$ reduces to $p$ and $0 . R * p$ reduces to $0 . R$ and $1 . R * p$ reduces to $p$.

One can check that $1_{R} \cdot p$ reduces to $p$.

Now we state the propositions:

(4) Let us consider an integral domain $R$, a polynomial $p$ over $R$, and a non zero element $a$ of $R$. Then $\operatorname{deg}(a \cdot p)=\operatorname{deg} p$.

(5) Let us consider an integral domain $R$, a polynomial $p$ over $R$, and an element $a$ of $R$. Then $\operatorname{LC}(a \cdot p)=a \cdot \operatorname{LC} p$.

(6) Let us consider an integral domain $R$, and an element $a$ of $R$. Then $\mathrm{LC}(a \uparrow R)=a$. The theorem is a consequence of (5).

(7) Let us consider an integral domain $R$, a polynomial $p$ over $R$, and elements $v, x$ of $R$. Then eval $(v \cdot p, x)=v \cdot \operatorname{eval}(p, x)$. The theorem is a consequence of (4).

(8) Let us consider a ring $R$, and elements $a, b$ of $R$. Then $\operatorname{eval}(a \nmid R, b)=a$.

Let $R$ be an integral domain and $p, q$ be monic polynomials over $R$. Let us note that $p * q$ is monic.

Let $a$ be an element of $R$ and $k$ be a natural number. One can check that $(\operatorname{rpoly}(1, a))^{k}$ is non zero and monic.

Now we state the propositions:

(9) Let us consider a non degenerated ring $R$, an element $a$ of $R$, and a non zero element $k$ of $\mathbb{N}$. Then $\operatorname{LC} \operatorname{rpoly}(k, a)=1_{R}$.

(10) Let us consider a non degenerated, well unital, non empty double loop structure $R$, and an element $a$ of $R$. Then $\left\langle-a, 1_{R}\right\rangle=\operatorname{rpoly}(1, a)$.

(11) Let us consider an integral domain $R$, a polynomial $p$ over $R$, and an element $x$ of $R$. Then eval $(p, x)=0_{R}$ if and only if $\operatorname{rpoly}(1, x) \mid p$. 
(12) Let us consider an integral domain $F$, polynomials $p, q$ over $F$, and an element $a$ of $F$. Suppose $\operatorname{rpoly}(1, a) \mid p * q$. Then

(i) $\operatorname{rpoly}(1, a) \mid p$, or

(ii) $\operatorname{rpoly}(1, a) \mid q$.

The theorem is a consequence of (11).

(13) Let us consider an integral domain $R$, a polynomial $p$ over $R$, and a non zero polynomial $q$ over $R$. If $p \mid q$, then $\operatorname{deg} p \leqslant \operatorname{deg} q$.

(14) Let us consider a non degenerated commutative ring $R$, a polynomial $q$ over $R$, a non zero polynomial $p$ over $R$, and a non zero element $b$ of $R$. If $q \mid p$, then $q \mid b \cdot p$.

(15) Let us consider a field $F$, a polynomial $q$ over $F$, a non zero polynomial $p$ over $F$, and a non zero element $b$ of $F$. Then $q \mid p$ if and only if $q \mid b \cdot p$. The theorem is a consequence of (14).

Let us consider an integral domain $R$, a non zero polynomial $p$ over $R$, an element $a$ of $R$, and a non zero element $b$ of $R$. Now we state the propositions:

(16) $\operatorname{rpoly}(1, a) \mid p$ if and only if $\operatorname{rpoly}(1, a) \mid b \cdot p$. The theorem is a consequence of (11), (7), and (14).

(17) $(\operatorname{rpoly}(1, a))^{n} \mid p$ if and only if $(\operatorname{rpoly}(1, a))^{n} \mid b \cdot p$.

Proof: Define $\mathcal{P}$ [natural number] $\equiv$ if $(\operatorname{rpoly}(1, a))^{\$_{1}} \mid b \cdot p$, then $(\operatorname{rpoly}(1, a))^{\$_{1}} \mid p$. For every natural number $k, \mathcal{P}[k]$.

Let $R$ be an integral domain, $p$ be a non zero polynomial over $R$, and $b$ be a non zero element of $R$. Let us note that $b \cdot p$ is non zero.

\section{On Roots of Polynomials}

Let $R$ be a non degenerated ring. One can check that 1.R and has not roots.

Let $a$ be a non zero element of $R$. One can verify that $a \uparrow R$ and has not roots and every polynomial over $R$ which is non zero and has roots is also non constant and every polynomial over $R$ which and has not roots is also non zero.

Let $a$ be an element of $R$. One can check that $\operatorname{rpoly}(1, a)$ is non zero and has roots and there exists a polynomial over $R$ which is non zero and has not roots and there exists a polynomial over $R$ which is non zero and has roots.

Let $R$ be an integral domain, $p$ be a polynomial over $R$ with non roots, and $a$ be a non zero element of $R$. Let us note that $a \cdot p$ and has not roots.

Let $p$ be a polynomial over $R$ with roots and $a$ be an element of $R$. Note that $a \cdot p$ has roots.

Let $R$ be a non degenerated commutative ring and $q$ be a polynomial over $R$. One can verify that $p * q$ has roots. 
Let $R$ be an integral domain and $p, q$ be polynomials over $R$ with non roots. One can check that $p * q$ and has not roots.

Let $R$ be a non degenerated commutative ring, $a$ be an element of $R$, and $k$ be a non zero element of $\mathbb{N}$. Let us note that $\operatorname{rpoly}(k, a)$ is non constant and monic and has roots.

Let $R$ be a non degenerated ring. Let us observe that there exists a polynomial over $R$ which is non constant and monic.

Let $R$ be an integral domain, $a$ be an element of $R, k$ be a non zero natural number, and $n$ be a non zero element of $\mathbb{N}$. Note that $(\operatorname{rpoly}(n, a))^{k}$ is non constant and monic and has roots.

Let $R$ be a ring and $p$ be a polynomial over $R$ with roots. Note that $\operatorname{Roots}(p)$ is non empty.

Let $R$ be a non degenerated ring and $p$ be a polynomial over $R$ with non roots. Let us observe that $\operatorname{Roots}(p)$ is empty.

Let $R$ be an integral domain. One can check that there exists a polynomial over $R$ which is monic and has roots and there exists a polynomial over $R$ which is monic and has not roots.

Now we state the propositions:

(18) Let us consider a non degenerated ring $R$, and an element $a$ of $R$. Then $\operatorname{Roots}(\operatorname{rpoly}(1, a))=\{a\}$.

(19) Let us consider an integral domain $F$, a polynomial $p$ over $F$, and a non zero element $b$ of $F$. Then $\operatorname{Roots}(b \cdot p)=\operatorname{Roots}(p)$. The theorem is a consequence of $(7)$.

(20) There exist polynomials $p, q$ over $\mathbb{Z} / 6$ such that $\operatorname{Roots}(p * q) \nsubseteq \operatorname{Roots}(p) \cup$ $\operatorname{Roots}(q)$.

(21) Let us consider an integral domain $R$, and elements $a, b$ of $R$. Then $\operatorname{rpoly}(1, a) \mid \operatorname{rpoly}(1, b)$ if and only if $a=b$. The theorem is a consequence of (18).

(22) Let us consider an integral domain $R$, and a non zero polynomial $p$ over $R$. Then $\overline{\overline{\operatorname{Roots}(p)}} \leqslant \operatorname{deg} p$.

\section{More about Bags}

Let $X$ be a non empty set and $B$ be a bag of $X$. We introduce the notation $\overline{\bar{B}}$ as a synonym of $\sum B$.

Observe that there exists a bag of $X$ which is zero and there exists a bag of $X$ which is non zero.

Let $b_{1}$ be a bag of $X$ and $b_{2}$ be a bag of $X$. One can check that $b_{1}+b_{2}$ is $X$-defined and $b_{1}+b_{2}$ is total. 
Let us consider a non empty set $X$ and a bag $b$ of $X$. Now we state the propositions:

(23) $\overline{\bar{b}}=0$ if and only if $\operatorname{support} b=\emptyset$.

(24) $b$ is zero if and only if support $b=\emptyset$.

(25) $b$ is zero if and only if $\operatorname{rng} b=\{0\}$.

Let $X$ be a non empty set, $b_{1}$ be a non zero bag of $X$, and $b_{2}$ be a bag of $X$. One can check that $b_{1}+b_{2}$ is non zero.

(26) Let us consider a non empty set $X$, a bag $b$ of $X$, and an element $x$ of $X$. Suppose support $b=\{x\}$. Then $b=(\{x\}, b(x))$-bag.

(27) Let us consider a non empty set $X$, a non empty bag $b$ of $X$, and an element $x$ of $X$. Then support $b=\{x\}$ if and only if $b=(\{x\}, b(x))$-bag and $b(x) \neq 0$. The theorem is a consequence of $(26)$.

Let $X$ be a set and $S$ be a finite subset of $X$. The functor $\operatorname{Bag}(S)$ yielding a bag of $X$ is defined by the term

(Def. 1) (S, 1) -bag.

Let $X$ be a non empty set and $S$ be a non empty, finite subset of $X$. Observe that $\operatorname{Bag}(S)$ is non zero.

Let $b$ be a bag of $X$ and $a$ be an element of $X$. The functor $b \backslash a$ yielding a bag of $X$ is defined by the term

(Def. 2$) \quad b+\cdot(a, 0)$.

Let us consider a non empty set $X$, a bag $b$ of $X$, and an element $a$ of $X$. Now we state the propositions:

(28) $b \backslash a=b$ if and only if $a \notin \operatorname{support} b$.

(29) $\operatorname{support}(b \backslash a)=\operatorname{support} b \backslash\{a\}$.

(30) $\quad(b \backslash a)+(\{a\}, b(a))-\mathrm{bag}=b$.

(31) Let us consider a non empty set $X$, an element $a$ of $X$, and an element $n$ of $\mathbb{N}$. Then $\overline{\overline{(\{a\}, n)-\mathrm{bag}}}=n$. The theorem is a consequence of (23).

\section{On Multiple Roots of Polynomials}

Let $R$ be an integral domain and $p$ be a non zero polynomial over $R$ with roots. One can verify that $\operatorname{BRoots}(p)$ is non zero.

Now we state the propositions:

(32) Let us consider a non degenerated commutative ring $R$, a non zero polynomial $p$ over $R$, and an element $a$ of $R$. Then multiplicity $(p, a)=0$ if and only if $\operatorname{rpoly}(1, a) \nmid p$. 
(33) Let us consider an integral domain $R$, a non zero polynomial $p$ over $R$, and an element $a$ of $R$. Then multiplicity $(p, a)=n$ if and only if $(\operatorname{rpoly}(1, a))^{n} \mid p$ and $(\operatorname{rpoly}(1, a))^{n+1} \nmid p$. The theorem is a consequence of (10).

(34) Let us consider an integral domain $R$, and an element $a$ of $R$. Then multiplicity $(\operatorname{rpoly}(1, a), a)=1$. The theorem is a consequence of $(13)$ and (33).

(35) Let us consider an integral domain $R$, and elements $a, b$ of $R$. If $b \neq a$, then multiplicity $(\operatorname{rpoly}(1, a), b)=0$. The theorem is a consequence of $(21)$ and (32).

(36) Let us consider an integral domain $R$, a non zero polynomial $p$ over $R$, a non zero element $b$ of $R$, and an element $a$ of $R$. Then multiplicity $(p, a)=$ multiplicity $(b \cdot p, a)$. The theorem is a consequence of (33), (14), and (17).

(37) Let us consider an integral domain $R$, a non zero polynomial $p$ over $R$, and a non zero element $b$ of $R$. Then $\operatorname{BRoots}(b \cdot p)=\operatorname{BRoots}(p)$. The theorem is a consequence of (36).

(38) Let us consider an integral domain $R$, and a non zero polynomial $p$ over $R$ without roots. Then $\operatorname{BRoots}(p)=\operatorname{EmptyBag}($ the carrier of $R$ ).

(39) Let us consider an integral domain $R$, and a non zero element $a$ of $R$. Then $\overline{\overline{\operatorname{BRoots}(a\lceil R)}}=0$. The theorem is a consequence of (23).

(40) Let us consider an integral domain $R$, and an element $a$ of $R$. Then $\overline{\overline{\text { BRoots}(\operatorname{rpoly}(1, a))}}=1$. The theorem is a consequence of $(10)$.

(41) Let us consider an integral domain $R$, and non zero polynomials $p, q$ over $R$. Then $\overline{\overline{\operatorname{BRoots}(p * q)}}=\overline{\overline{\operatorname{BRoots}(p)}}+\overline{\overline{\operatorname{BRoots}(q)}}$.

(42) Let us consider an integral domain $R$, and a non zero polynomial $p$ over $R$. Then $\overline{\overline{\operatorname{BRoots}(p)}} \leqslant \operatorname{deg} p$.

Proof: Define $\mathcal{P}$ [natural number] $\equiv$ for every non zero polynomial $p$ over $R$ such that $\operatorname{deg} p=\$_{1}$ holds $\overline{\overline{\operatorname{BRoots}(p)}} \leqslant \operatorname{deg} p$. $\mathcal{P}[0]$. For every natural number $k, \mathcal{P}[k]$. 


\section{The Polynomial $X^{n}+1$}

Let $R$ be a unital, non empty double loop structure and $n$ be a natural number. The functor $\operatorname{npoly}(R, n)$ yielding a sequence of $R$ is defined by the term

(Def. 3) $\quad$ o.R+· $\left[0 \longmapsto 1_{R}, n \longmapsto 1_{R}\right]$.

One can check that $\operatorname{npoly}(R, n)$ is finite-Support and $\operatorname{npoly}(R, n)$ is non zero.

Let us consider a unital, non degenerated double loop structure $R$. Now we state the propositions:

(43) $\operatorname{deg} \operatorname{npoly}(R, n)=n$.

(44) $\operatorname{LC} \operatorname{npoly}(R, n)=1_{R}$.

(45) Let us consider a non degenerated ring $R$, and an element $x$ of $R$. Then $\operatorname{eval}(\operatorname{npoly}(R, 0), x)=1_{R}$.

(46) Let us consider a non degenerated ring $R$, a non zero natural number $n$, and an element $x$ of $R$. Then eval(npoly $(R, n), x)=x^{n}+1_{R}$.

Proof: Set $q=\operatorname{npoly}(R, n)$. Consider $F$ being a finite sequence of elements of $R$ such that $\operatorname{eval}(q, x)=\sum F$ and len $F=\operatorname{len} q$ and for every element $j$ of $\mathbb{N}$ such that $j \in \operatorname{dom} F$ holds $F(j)=q\left(j-^{\prime} 1\right) \cdot \operatorname{power}_{R}\left(x, j-{ }^{\prime} 1\right)$. Consider $f_{1}$ being a sequence of the carrier of $R$ such that $\sum F=f_{1}(\operatorname{len} F)$ and $f_{1}(0)=0_{R}$ and for every natural number $j$ and for every element $v$ of $R$ such that $j<$ len $F$ and $v=F(j+1)$ holds $f_{1}(j+1)=f_{1}(j)+v$. Define $\mathcal{P}$ [element of $\mathbb{N}] \equiv \$_{1}=0$ and $f_{1}\left(\$_{1}\right)=0_{R}$ or $0<\$_{1}<$ len $F$ and $f_{1}\left(\$_{1}\right)=1_{R}$ or $\$_{1}=$ len $F$ and $f_{1}\left(\$_{1}\right)=x^{n}+1_{R}$. For every element $j$ of $\mathbb{N}$ such that $0 \leqslant j \leqslant \operatorname{len} F$ holds $\mathcal{P}[j]$.

(47) Let us consider an even natural number $n$, and an element $x$ of $\mathbb{R}_{\mathrm{F}}$. Then eval $\left(\operatorname{npoly}\left(\mathbb{R}_{\mathrm{F}}, n\right), x\right)>0_{\mathbb{R}_{\mathrm{F}}}$. The theorem is a consequence of (45), (1), and (46).

(48) Let us consider an odd natural number $n$. Then eval(npoly $\left.\left(\mathbb{R}_{F}, n\right),-1_{\mathbb{R}_{F}}\right)$ $=0_{\mathbb{R}_{\mathrm{F}}}$. The theorem is a consequence of (46).

(49) $\operatorname{eval}\left(\operatorname{npoly}(\mathbb{Z} / 2,2), 1_{\mathbb{Z} / 2}\right)=0_{\mathbb{Z} / 2}$. The theorem is a consequence of $(46)$ and $(2)$.

Let $n$ be an even natural number. Let us note that npoly $\left(\mathbb{R}_{\mathrm{F}}, n\right)$ and has not roots.

Let $n$ be an odd natural number. Observe that $\operatorname{npoly}\left(\mathbb{R}_{\mathrm{F}}, n\right)$ has roots and $\operatorname{npoly}(\mathbb{Z} / 2,2)$ has roots. 


\section{The Polynomials $\left(x-a_{1}\right) *\left(x-a_{2}\right) * \ldots *\left(x-a_{n}\right)$}

Let $R$ be a ring.

A product of linear polynomials of $R$ is a polynomial over $R$ and is defined by

(Def. 4) there exists a non empty finite sequence $F$ of elements of PolyRing $(R)$ such that $i t=\prod F$ and for every natural number $i$ such that $i \in \operatorname{dom} F$ there exists an element $a$ of $R$ such that $F(i)=\operatorname{rpoly}(1, a)$.

Let $R$ be an integral domain. One can verify that every product of linear polynomials of $R$ is non constant and monic and has roots.

Now we state the propositions:

(50) Let us consider an integral domain $R$, and a product of linear polynomials $p$ of $R$. Then $\operatorname{LC} p=1_{R}$.

(51) Let us consider an integral domain $R$, and an element $a$ of $R$. Then $\operatorname{rpoly}(1, a)$ is a product of linear polynomials of $R$.

(52) Let us consider an integral domain $R$, and products of linear polynomials $p, q$ of $R$. Then $p * q$ is a product of linear polynomials of $R$.

Let $R$ be an integral domain and $B$ be a non zero bag of the carrier of $R$.

A product of linear polynomials of $R$ and $B$ is a product of linear polynomials of $R$ and is defined by

(Def. 5) $\operatorname{deg} i t=\overline{\bar{B}}$ and for every element $a$ of $R$, multiplicity $(i t, a)=B(a)$.

Let us consider an integral domain $R$, a non zero bag $B$ of the carrier of $R$, a product of linear polynomials $p$ of $R$ and $B$, and an element $a$ of $R$. Now we state the propositions:

(53) If $a \in \operatorname{support} B$, then $\operatorname{eval}(p, a)=0_{R}$. The theorem is a consequence of (11).

(i) $(\operatorname{rpoly}(1, a))^{B(a)} \mid p$, and

(ii) $(\operatorname{rpoly}(1, a))^{B(a)+1} \nmid p$.

The theorem is a consequence of (33).

Let us consider an integral domain $R$, a non zero bag $B$ of the carrier of $R$, and a product of linear polynomials $p$ of $R$ and $B$. Now we state the propositions:

(55) $\operatorname{BRoots}(p)=B$.

(56) $\operatorname{deg} p=\overline{\overline{\operatorname{BRoots}(p)}}$. The theorem is a consequence of (55).

(57) Let us consider an integral domain $R$, and an element $a$ of $R$. Then $\operatorname{rpoly}(1, a)$ is a product of linear polynomials of $R$ and $\operatorname{Bag}(\{a\})$. The theorem is a consequence of (51), (34), and (35).

(58) Let us consider an integral domain $R$, non zero bags $B_{1}, B_{2}$ of the carrier of $R$, a product of linear polynomials $p$ of $R$ and $B_{1}$, and a product of linear 
polynomials $q$ of $R$ and $B_{2}$. Then $p * q$ is a product of linear polynomials of $R$ and $B_{1}+B_{2}$. The theorem is a consequence of (52), (56), and (55).

(59) Let us consider an integral domain $R$. Then every product of linear polynomials of $R$ is a product of linear polynomials of $R$ and $\operatorname{BRoots}(p)$. Proof: Define $\mathcal{P}$ [natural number] $\equiv$ for every product of linear polynomials $p$ of $R$ such that $\operatorname{deg} p=\$_{1}$ holds $p$ is a product of linear polynomials of $R$ and $\operatorname{BRoots}(p)$. $\mathcal{P}[1]$. For every natural number $k$ such that $k \geqslant 1$ holds $\mathcal{P}[k]$.

Let $R$ be an integral domain and $S$ be a non empty, finite subset of $R$.

A product of linear polynomials of $R$ and $S$ is a product of linear polynomials of $R$ and $\operatorname{Bag}(S)$. Now we state the proposition:

(60) Let us consider an integral domain $R$, a non empty, finite subset $S$ of $R$, and a product of linear polynomials $p$ of $R$ and $S$. Then $\operatorname{deg} p=\overline{\bar{S}}$.

Let us consider an integral domain $R$, a non empty, finite subset $S$ of $R$, a product of linear polynomials $p$ of $R$ and $S$, and an element $a$ of $R$. Now we state the propositions:

(61) If $a \in S$, then $\operatorname{rpoly}(1, a) \mid p$ and $(\operatorname{rpoly}(1, a))^{2} \nmid p$. The theorem is a consequence of (54).

(62) If $a \in S$, then $\operatorname{eval}(p, a)=0_{R}$. The theorem is a consequence of $(61)$.

(63) Let us consider an integral domain $R$, a non empty, finite subset $S$ of $R$, and a product of linear polynomials $p$ of $R$ and $S$. Then $\operatorname{Roots}(p)=S$. The theorem is a consequence of (62), (22), and (60).

\section{MAIN THEOREMS}

Now we state the proposition:

(64) Let us consider an integral domain $R$, and a non zero polynomial $p$ over $R$ with roots. Then there exists a product of linear polynomials $q$ of $R$ and $\operatorname{BRoots}(p)$ and there exists a polynomial $r$ over $R$ with non roots such that $p=q * r$ and $\operatorname{Roots}(q)=\operatorname{Roots}(p)$.

Proof: Define $\mathcal{P}$ [natural number] $\equiv$ for every non zero polynomial $p$ over $R$ with roots such that $\operatorname{deg} p=\$_{1}$ there exists a product of linear polynomials $q$ of $R$ and $\operatorname{BRoots}(p)$ and there exists a polynomial $r$ over $R$ with non roots such that $p=q * r$ and $\operatorname{Roots}(q)=\operatorname{Roots}(p) . \mathcal{P}[1]$ by (11), [9, (1)], (51), [8, (23), (27), (24)]. For every natural number $k$ such that $1 \leqslant k$ holds $\mathcal{P}[k]$. Consider $d$ being a natural number such that $\operatorname{deg} p=d$.

Let us consider an integral domain $R$ and a non zero polynomial $p$ over $R$. 
(65) $\overline{\overline{\operatorname{Roots}(p)}} \leqslant \overline{\overline{\operatorname{BRoots}(p)}}$. The theorem is a consequence of $(64),(56),(55)$, $(22)$, and (38).

(66) $\overline{\overline{\operatorname{BRoots}(p)}}=\operatorname{deg} p$ if and only if there exists an element $a$ of $R$ and there exists a product of linear polynomials $q$ of $R$ such that $p=a \cdot q$. The theorem is a consequence of (64), (56), (55), (59), (4), (37), and (38).

Now we state the proposition:

(67) Let us consider an integral domain $R$, and polynomials $p, q$ over $R$. Suppose there exists a subset $S$ of $R$ such that $\overline{\bar{S}}=\max (\operatorname{deg} p, \operatorname{deg} q)+1$ and for every element $a$ of $R$ such that $a \in S \operatorname{holds} \operatorname{eval}(p, a)=\operatorname{eval}(q, a)$. Then $p=q$. The theorem is a consequence of (22).

Let $F$ be an algebraic closed field. Note that every non constant polynomial over $F$ has roots and $\mathbb{R}_{F}$ is non algebraic closed and every finite integral domain is non algebraic closed and every ring which is algebraic closed is also almost right invertible.

Now we state the propositions:

(68) Let us consider an algebraic closed field $F$, and a non constant polynomial $p$ over $F$. Then there exists an element $a$ of $F$ and there exists a product of linear polynomials $q$ of $F$ and $\operatorname{BRoots}(p)$ such that $a \cdot q=p$. The theorem is a consequence of (64).

(69) Let us consider an algebraic closed field $F$. Then every non constant, monic polynomial over $F$ is a product of linear polynomials of $F$ and $\operatorname{BRoots}(p)$. The theorem is a consequence of (68).

(70) Let us consider a field $F$. Then $F$ is algebraic closed if and only if every non constant, monic polynomial over $F$ is a product of linear polynomials of $F$. The theorem is a consequence of (69).

\section{REFERENCES}

[1] Grzegorz Bancerek, Czesław Byliński, Adam Grabowski, Artur Korniłowicz, Roman Matuszewski, Adam Naumowicz, Karol Pąk, and Josef Urban. Mizar: State-of-the-art and beyond. In Manfred Kerber, Jacques Carette, Cezary Kaliszyk, Florian Rabe, and Volker Sorge, editors, Intelligent Computer Mathematics, volume 9150 of Lecture Notes in Computer Science, pages 261-279. Springer International Publishing, 2015. ISBN 978-3319-20614-1. doi:10.1007/978-3-319-20615-8_17.

[2] Adam Grabowski, Artur Korniłowicz, and Adam Naumowicz. Four decades of Mizar. Journal of Automated Reasoning, 55(3):191-198, 2015. doi 10.1007/s10817-015-9345-1.

[3] Adam Grabowski, Artur Korniłowicz, and Christoph Schwarzweller. On algebraic hierarchies in mathematical repository of Mizar. In M. Ganzha, L. Maciaszek, and M. Paprzycki, editors, Proceedings of the 2016 Federated Conference on Computer Science and Information Systems (FedCSIS), volume 8 of Annals of Computer Science and Information Systems, pages 363-371, 2016. doi $10.15439 / 2016 \mathrm{~F} 520$.

[4] H. Heuser. Lehrbuch der Analysis. B.G. Teubner Stuttgart, 1990.

[5] Nathan Jacobson. Basic Algebra I. 2nd edition. Dover Publications Inc., 2009. 
[6] Heinz Lüneburg. Gruppen, Ringe, Körper: Die grundlegenden Strukturen der Algebra. Oldenbourg Verlag, 1990.

[7] Knut Radbruch. Algebra I. Lecture Notes, University of Kaiserslautern, Germany, 1991.

[8] Christoph Schwarzweller and Agnieszka Rowińska-Schwarzweller. Schur's theorem on the stability of networks. Formalized Mathematics, 14(4):135-142, 2006. doi:10.2478/v10037006-0017-9

[9] Christoph Schwarzweller, Artur Korniłowicz, and Agnieszka Rowińska-Schwarzweller. Some algebraic properties of polynomial rings. Formalized Mathematics, 24(3):227-237, 2016. doi $10.1515 /$ forma-2016-0019.

Received August 30, 2017

The English version of this volume of Formalized Mathematics was financed under agreement 548/P-DUN/2016 with the funds from the Polish Minister of Science and Higher Education for the dissemination of science. 